\title{
Polyanisotropic magnetoelectric coupling in an electrically controlled molecular spin qubit
}

Jérôme Robert, ${ }^{\S \dagger}$ Nathalie Parizel, ${ }^{\dagger}$ Philippe Turek, ${ }^{\dagger}$ Athanassios K. Boudalis ${ }^{\dagger} *$

${ }^{\dagger}$ Institut de Chimie de Strasbourg (UMR 7177, CNRS-Unistra), Université de Strasbourg, 4 rue Blaise Pascal, CS 90032, F-67081 Strasbourg, France.

E-mail: bountalis@unistra.fr

${ }^{\S}$ Sorbonne Université, CNRS, Laboratoire Jean Perrin, LJP, F-75005 Paris, France.

\begin{abstract}
Two molecular spin qubits are studied with pulsed Electron Paramagnetic Resonance (EPR) spectroscopy under electric fields to assess their magnetoelectric (ME) couplings and electric spin control. $\left[\mathrm{Fe}_{3} \mathrm{O}(\mathrm{PhCOO})_{6}(\mathrm{py})_{3}\right] \mathrm{ClO}_{4}$ ·py $\left(\mathbf{F e}_{3}\right)$ is characterized by strong Dzyaloshinskii-Moriya interactions (DMI) which induce important magnetoanisotropy, whereas the DMI in $\left[\mathrm{Cr}_{3} \mathrm{O}(\mathrm{PhCOO})_{6}(\mathrm{py})_{3}\right] \mathrm{ClO}_{4} \cdot 0.5 \mathrm{py}\left(\mathbf{C r}_{3}\right)$ is $1-2$ orders of magnitude weaker. $\mathbf{F e}_{3}$ is observed to demonstrate a clear ME effect whose intensity shows an unprecedented dependence on the molecular orientation within the electric field $\mathbf{E}$ (electroanisotropy) and on the relative orientations of the molecular z-axis, the Zeeman field $\mathbf{B}_{0}$ and $\mathbf{E}$ (magnetoelectric anisotropy). The electric control in $\mathbf{F} \mathbf{e}_{3}$ is shown to be coherent and the ME effect exhibits complex dynamics characterized by saturation and oscillatory effects. On the other hand, $\mathbf{C r}_{3}$ exhibits no discernible ME effect, which correlates well with its negligible DMI.
\end{abstract}




\section{Introduction}

Recent years have seen a surge in the search for materials that combine electric and magnetic properties; these are classified as "multiferroic" when they combine different types of electric and magnetic order and "magnetoelectric" when a coupling between their electric and magnetic properties allows the electric control of their magnetization and/or magnetic control of their polarization. ${ }^{1,2}$ This recent interest is fueled by their consideration, among others, as materials for new types of memories, or as electrically controlled spintronics elements, such as spin qubits. Regarding the latter aspect, electric control of magnetic quantum objects constitutes a key technological advantage, as it can lead to spatial and temporal resolutions unattainable by magnetic fields.

Different types of ME materials have been identified, with different mechanisms active in each case. The hyperfine Stark effect is operative in ${ }^{31} \mathrm{P}$ donor atoms in ${ }^{28} \mathrm{Si}^{3}$ and in the molecular nanomagnet (MNM) $\left[\mathrm{Tb}(\mathrm{pc})_{2}\right]$; $^{4,5}$ zero-field splitting (zfs) induces ME couplings in $\mathrm{ZnO}: \mathrm{Mn}^{\mathrm{II}}$ implants, ${ }^{6}$ the molecular $\mathrm{Cr}_{7} \mathrm{Mn}$ ring ${ }^{7}$ and nickelocene; ${ }^{8}$ magnetic exchange modulation was shown to occur in a $\mathrm{Mn}^{\mathrm{II}}$ radical helix under modulated E-fields ${ }^{9}$ and in the $\mathrm{Cu}{ }_{3}{ }_{3}$-saltag frustrated triangle under pulsed E-fields. ${ }^{7}$

Along these lines, we recently used continuous-wave EPR spectroscopy to demonstrate the ME coupling in the molecular spin triangle $\left[\mathrm{Fe}_{3} \mathrm{O}(\mathrm{PhCOO})_{6}(\mathrm{py})_{3}\right] \mathrm{ClO}_{4} \cdot \mathrm{py}\left(\mathbf{F e}_{3}\right.$, Figure 1), using static electric fields of up to $\sim 10^{7} \mathrm{Vm}^{-1}$ applied along the triangle plane. ${ }^{10}$ 


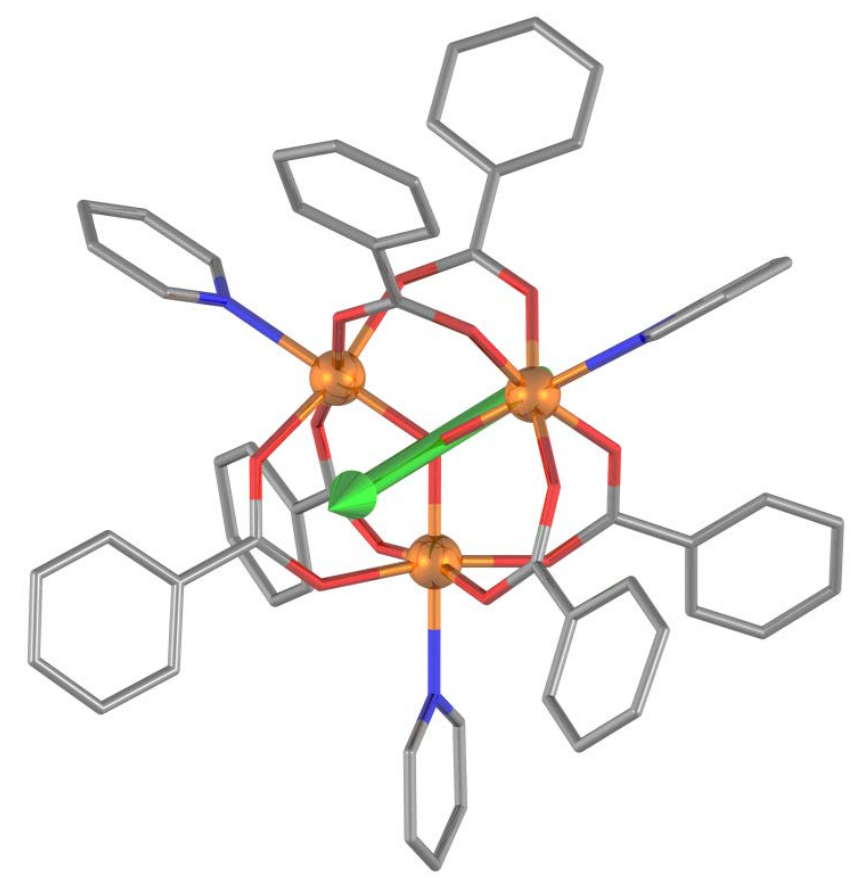

Figure 1. POV-Ray plot of the cation of $\mathbf{F e}_{3}$, showing the crystallographically imposed $C_{3}$ axis (green arrow). The three iron atoms (orange) are equivalent due to symmetry. Color key: carbon (grey), nitrogen (blue), oxygen (red). The structure of the cation of $\mathbf{C r}_{\mathbf{3}}$ is visually indistinguishable and is not presented separately.

We have been particularly interested in the ME coupling in spin triangles due to their proposed use as electrically controlled, slow-decoherence spin-chirality qubits. While MNMs have been considered as spin qubits for several years, ${ }^{11,12}$ a common stumbling block is their rapid decoherence. The strategies of isotopic engineering ${ }^{13}$ and of the use of nuclear, instead of electronic, spins (e.g. in $\left[\mathrm{Tb}(\mathrm{pc})_{2}\right]^{14}$ ) have yielded impressive improvements. However, the former solution can be prohibitively costly, and also restrictive as certain elements lack stable non-magnetic isotopes (e.g. $\mathrm{H}, \mathrm{N}, \mathrm{Cl}$ ); the latter has yielded phase memory times ${ }^{5,15,16}$ which are still significantly below the record of the isotopically-engineered $\left(\mathrm{Ph}_{4} \mathrm{P}-d_{20}\right)_{2}\left[\mathrm{~V}\left(\mathrm{C}_{8} \mathrm{~S}_{8}\right)_{3}\right] .{ }^{13}$ An innovative solution particularly adapted to MNMs with their large Hilbert spaces, proposes to assign the qubit's logical values to an appropriate Hilbert subspace which would be insensitive to hyperfine interactions, ${ }^{17}$ thus embedding coherence protection 
in the qubit design. In particular, it was proposed to use spin triangles characterized by a chiral spin texture, and to encode the qubit states on the eigenvalues of their scalar spin chirality operator, thus ensuring protection from hyperfine-induced decoherence for $\mathbf{B}_{0} \| \mathrm{z} .{ }^{18,19}$ Moreover, due to their noncentrosymmetric structures, the triangles’ spins were postulated to couple with external electric fields, thus allowing their direct electric manipulation, including their long-range coupling. ${ }^{20}$ This $\mathrm{ME}$ coupling hypothesis was confirmed by our single-crystal studies on $\mathbf{F e}_{3}{ }^{10}$ and by pulsed EPR studies on a $\mathrm{Cu}_{3}{ }_{3}$ triangle. ${ }^{7}$ However, whereas symmetry rules can generally predict when ME coupling is allowed, they cannot predict whether it will be sufficiently strong to actually be observable. Attempts have been made for the $a b$ initio prediction of its strength, ${ }^{21}$ but experimental studies are still rare and not systematic.

Here we investigate the factors that determine the strength of ME coupling in two molecular spin qubits we have previously studied, ${ }^{22}$ the magnetoelectric $\mathbf{F e}_{3}{ }^{10,23}$ and its $\mathrm{Cr}^{\text {III }}$ analogue $\left[\mathrm{Cr}_{3} \mathrm{O}(\mathrm{PhCOO})_{6}(\mathrm{py})_{3}\right] \mathrm{ClO}_{4} \cdot 0.5 \mathrm{py}\left(\mathbf{C r}_{3}\right)^{24}$, whose close structural and chemical features make them an ideal couple for this purpose. We discover coherent electric control of the magnetization and unprecedented features regarding the anisotropy and dynamic nature of the ME coupling. In addition, we propose a phenomenological identification of the factors that influence its strength.

\section{Experimental}

Complexes $\mathbf{F e}_{3}$ and $\mathbf{C r}_{3}$ were prepared as previously described. ${ }^{10,24}$ Experiments were carried out on fresh py- $\mathrm{D}_{5}$ solutions (2.3 mM), prepared by slight heating to accelerate dissolution and the exchange of axial py with py- $d_{5}$. Pulsed X-band $(9.75 \mathrm{GHz})$ EPR spectra were collected on the frozen solutions $(4.40 \mathrm{~K})$ on a Bruker ELEXSYS E580 spectrometer, fitted with an upgraded ESP1010 microwave bridge and a Bruker EN 4118X-MD4 pulse-ENDOR resonator. For low-temperature experiments the resonator was fitted in an Oxford CF935 dynamic continuous flow cryostat and the temperature was regulated with an Oxford Mercury iTC. Prior to experiments, the samples were routinely characterized 
by field-sweep echo-detected (FSED) spectra using the intensity of the Hahn echo ( $\pi / 2-\tau-\pi-\tau$-echo) under varying fields.

Pulsed electric fields were applied by Ag wire electrodes (99.99\%, Goodfellow), which were immersed vertically into the solutions, which were subsequently frozen. The electrodes were connected to an external power supply through a coaxial cable. The electric pulses were generated with a Direct Energy Inc. PVM-4210 dual output high voltage pulse generator, capable of generating up to $950 \mathrm{~V}$ pulses with less than $15 \mathrm{~ns}$ rise and fall times and at up to $20 \mathrm{kHz}$ frequencies. The frequency of the electric pulses was defined by the Shot Repetition Time (SRT) of the experiment, which was 2 ms and corresponded to a $500 \mathrm{~Hz}$ frequency. Synchronization with the microwave pulses was achieved using the TTL signal from a spare port of the PDCH board of the SpecJet device in the ELEXSYS, which was fed to the trigger port of the high voltage power supply. The pulse patterns were routinely programmed in PulseSPEL syntax, specifically using the reserved port U1 to trigger the electrical pulses.

Ac susceptibility experiments at $1111 \mathrm{~Hz}$ were carried out on a Quantum Design PPMS magnetometer using a $10 \mathrm{G}$ oscillating field. Spin expectation values $\left\langle S_{k}\right\rangle(k=x, y, z)$ were calculated with Matlab with custom-made routines using functions from the Easyspin ${ }^{25}$ toolbox.

\section{Results}

The basic magnetic structure of spin triangles is characterized by an antiferromagnetic HeisenbergDirac-van Vleck (HDvV, isotropic) exchange term characterized by an isosceles symmetry breaking $\left(J_{12}=J_{13}=J, J_{23}=J^{\prime}, J \neq J^{\prime}\right)$, and by a Dzyaloshinskii-Moriya (DM, antisymmetric) exchange term. The spin Hamiltonian that describes their magnetic properties, including their Zeeman interaction to a magnetic field, is written as:

$$
H=-2 J\left(\hat{\mathbf{S}}_{1} \hat{\mathbf{S}}_{2}+\hat{\mathbf{S}}_{1} \hat{\mathbf{S}}_{3}\right)-2 J \hat{\mathbf{S}}_{2} \hat{\mathbf{S}}_{3}-2 \mathbf{G}\left(\hat{\mathbf{S}}_{1} \times \hat{\mathbf{S}}_{2}+\hat{\mathbf{S}}_{2} \times \hat{\mathbf{S}}_{3}+\hat{\mathbf{S}}_{3} \times \hat{\mathbf{S}}_{1}\right)+\mu_{B} \mathbf{H} \Sigma \mathbf{g}_{i} \hat{\mathbf{S}}_{i}
$$


where $\mathbf{G}$ is the pseudovector of the DM interaction.

The former term lifts the degeneracy of the low-lying $S_{T}=1 / 2$ states by an energy $\Delta \propto\left|J-J^{\prime}\right|$, in what is known as the "Magnetic Jahn-Teller Effect" (MJTE) ${ }^{26}$ and which has also been considered in a dynamic context of atomic vibrations. ${ }^{23,27-29}$ The latter term, apart from further increasing this energy, also induces an anisotropy of these states, most visible as a downfield shift of the $g_{\perp}$ resonance in the EPR spectra of such complexes. ${ }^{30-33}$

Table 1. Spin Hamiltonian parameters and effective $g$ values of $\mathbf{F e}_{3}$ and $\mathbf{C r}_{3}$, determined from solidstate studies (references ${ }^{23}$ and ${ }^{24}$, respectively). The values of the parameters refer to the formalism of Hamiltonian (1). Effective $g$ values in frozen pyridine solutions are taken from reference ${ }^{22}$.

\begin{tabular}{llllll}
\hline Complex & $J_{\mathrm{av}}\left(\mathrm{cm}^{-1}\right)^{a}$ & $|\Delta J|\left(\mathrm{cm}^{-1}\right)^{b}$ & $|\mathbf{G}|\left(\mathrm{cm}^{-1}\right)$ & $g_{\| \text {eff }}$ & $g_{\perp \text { eff }}$ \\
\hline $\mathbf{F e}_{3}$ & -21.6 & $5-6$ & $1.6-1.7$ & 2.00 & Powder: 1.3-1.0 (avg: 1.1) \\
& & & & Solution: $1.9-1.65$ \\
$\mathbf{C r}_{3}$ & -10.8 & 0.3 & 0.04 & 1.97 & Powder: 1.98-1.5 (avg: 1.8) \\
& & & & & Solution: 1.98-1.95
\end{tabular}
${ }^{a}\left(2 J+J^{\prime}\right) / 3 \cdot{ }^{b}\left|J-J^{\prime}\right|$.

The basic experiment that was implemented in these studies was the Hahn echo, which consists of a $\pi / 2$ and a $\pi$ microwave pulse separated by a delay $\tau$, giving rise to an echo at time $2 \tau$ (Figure $2 \mathrm{a}$ ). According to the method of Mims, ${ }^{34,35}$ electric field pulses interjected in the sequence can induce an additional dephasing of the spin packets, which is visible as a decrease of the echo intensity. Mims implemented electric pulses only after the $\pi$ pulse due to the long fall time of the voltage generator he used, but improvements in electronics have allowed modifications of the method and positioning of the electric pulse anywhere between the $\pi / 2$ pulse and the echo. ${ }^{3}$ 
In our studies we used two straight-wire electrodes to apply the electric field in the sample solution, which is well suited for solutions of molecular materials, despite the inhomogeneous electric field generated. ${ }^{7}$ Electrostatic simulations confirm that the net electric field has sufficient directionality for the detection of eventual electroanisotropies of these molecules, and that its strength is directly proportional to the applied voltage (see SI).

In addition, due to the nature of the EPR experiment, by properly selecting the magnetic field strength these studies also allowed us to discriminate for particular molecular orientations with in relation to the magnetic field. In particular, for any given magnetic field strength, only molecular populations with specific orientations come into resonance, and it is these molecules which undergo coherent spin manipulation by the Hahn echo sequence. Thus, our method allows to discriminate for molecular orientations with respect to either field, and to detect eventual anisotropies.
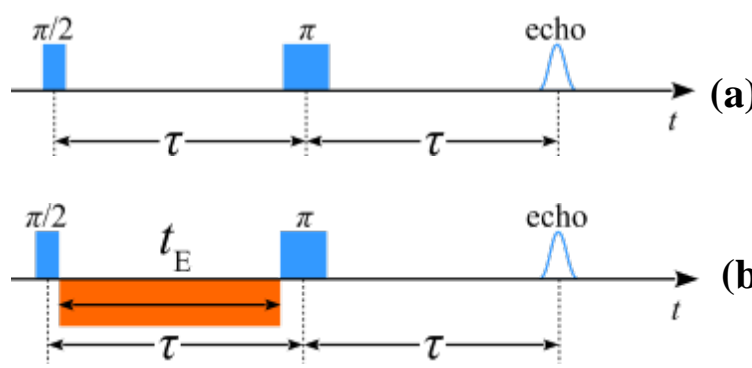

(b)

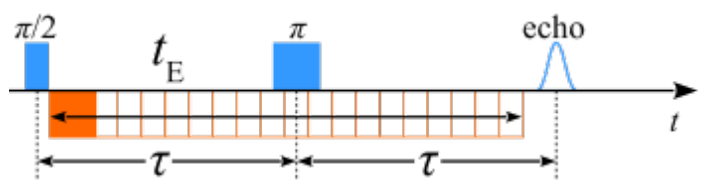

(c)
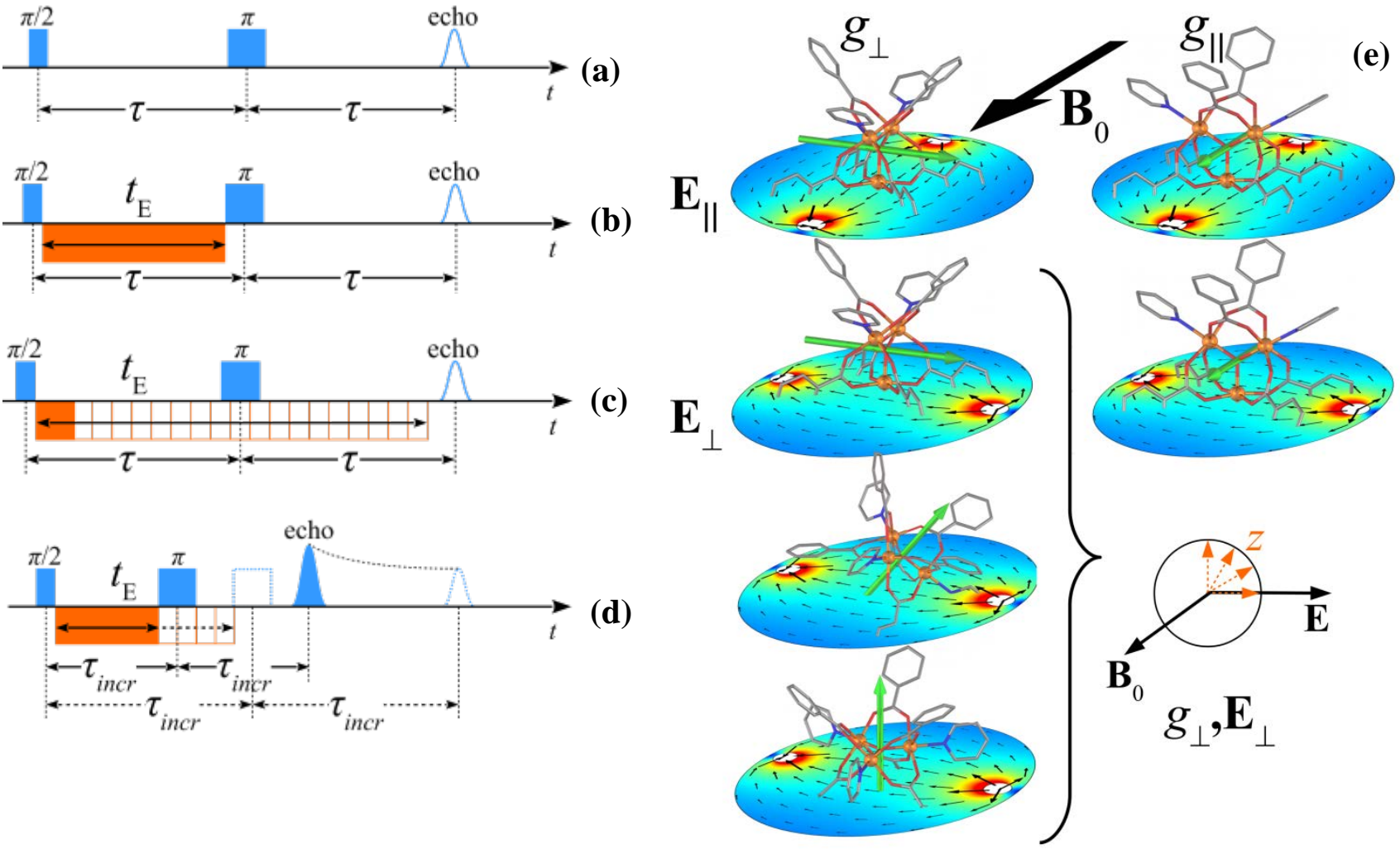

(e)

(d) 
Figure 2. Details of the experimental pulse sequences and geometry. (a)-(d). Pulse sequences used in this work, based on the standard Hahn echo. (e). Pictorial representation of the relative orientations of the molecular $z$-axis (green arrow), the Zeeman field $\left(\mathbf{B}_{0}\right)$ and the electric field (vector-field plot slices) in the limiting cases of the experiments. It is to be noted that the electric field indexes (\| or $\perp$ ) are in relation to $\mathbf{B}_{0}$ and not to the molecular z-axis. In the $g_{\|}$cases, the molecular orientation is uniquely defined with respect to $\mathbf{B}_{0}$, and consequently it is also so uniquely defined with respect to $\mathbf{E}$. Thus, for ( $\left.g_{\|}, \mathbf{E}_{\|}\right)$we have $\mathbf{E} \| z$, and for $\left(g_{\|}, \mathbf{E}_{\perp}\right)$ we have $\mathbf{E} \perp z$. In the $g_{\perp}$ cases, there are infinite equivalent orientations for which $\mathbf{B}_{0} \perp z$. Fortuitously, in the $\left(g_{\perp}, \mathbf{E}_{\|}\right)$case it holds that $\mathbf{E} \perp z$ for all of them, conserving their equivalence. However, in the $\left(g_{\perp}, \mathbf{E}_{\perp}\right)$ case (bottom left), each of these orientations has a different angle with respect to $\mathbf{E}$, thus their equivalence is destroyed due to the presence of that second field. In the figure we show three such indicative orientations (horizontal, $45^{\circ}$ and vertical) which are all normal to $\mathbf{B}_{0}$ and magnetically equivalent, but which exhibit different angles relative to $\mathbf{E}$.

Preliminary experiments were carried out on $\mathbf{F e}_{3}$ at magnetic fields corresponding to its $g_{\|}$value, with the electrodes oriented so that $\mathbf{E} \| \mathbf{B}_{0}\left(V_{\|}=950 \mathrm{~V},\left|\mathbf{E}_{\|}\right| \sim 2.9 \times 10^{5} \mathrm{Vm}^{-1}\right)$. The incremented electric pulses produced a clear suppression of the echo ( $40 \%$ of its initial intensity after $3 \mu \mathrm{s})$, thus confirming the previously observed ME coupling. Interested in assessing the effect of the molecular orientation, we repeated the experiment at the $g_{\perp}$ resonance $(\sim 411 \mathrm{mT})$, revealing several new phenomena (Figure 3). First, the decrease of the echo intensity was precipitous, being annihilated after a pulse of $~ 500$ ns. Second, beyond that pulse duration, the echoes became negative, indicating that the effect was strong enough to induce a partial reversal of the average magnetization. Third, oscillations of the echo intensity were observed, superimposed to its quasi-exponential decay. 

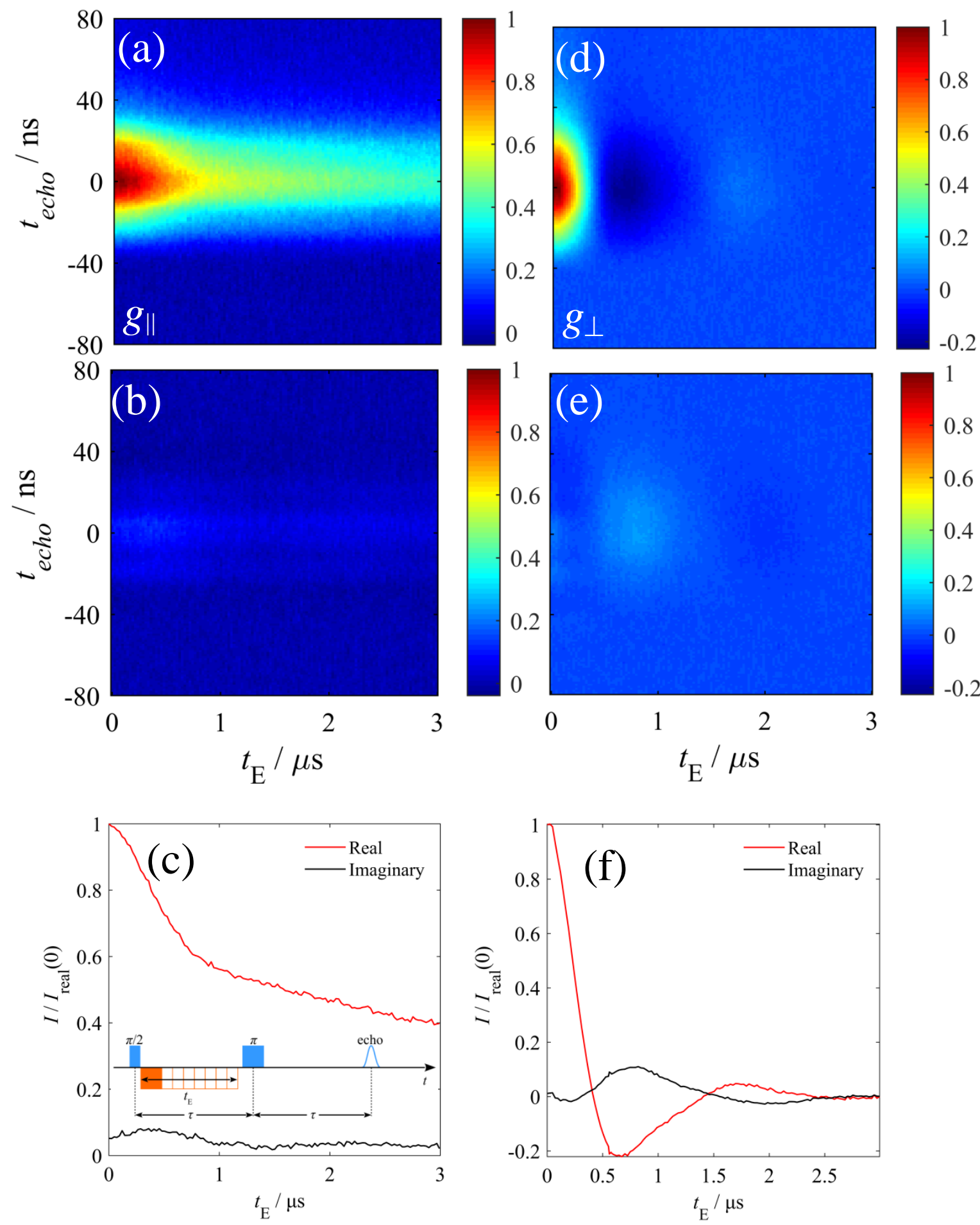

Figure 3. 2D plots of the Hahn echoes of $\mathbf{F e}_{3}(\tau=3 \mu \mathrm{s})$ as a function of the electric pulse duration, $t_{E}$. (a), (b) Real and imaginary parts at the $g_{\|}$resonance. (d), (e) Similar for the $g_{\perp}$ resonance. Panels (c) and (f) show the integrated intensities of the real and imaginary parts for the two resonance positions. 
These observations were refined through a systematic experimental protocol: (i) Field-swept echodetected (FSED) spectra ( $\tau=3 \mu \mathrm{s}$ ) were collected by applying electric pulses immediately after the end of the initial $\pi / 2$ pulse. (ii) After each FSED spectrum the pulse duration was incremented, so as to span the range $0<t_{E}<2 \tau$ ( $=6 \mu$ s) for the entire sequence, i.e. reaching right up to the echo at its longest duration. As has been previously argued, ${ }^{7}$ recovery of the echo intensity at $t_{E}=2 \tau$ demonstrates that the magnetizations are refocused when the electric pulse is applied over the defocusing and refocusing periods of the Hahn sequence, indicating a coherent electric manipulation of the spins. (iii) This set of scans was repeated for $\mathbf{E} \perp \mathbf{B}_{0}\left(\mathrm{E}_{\perp}\right)$ and $\mathbf{E} \| \mathbf{B}_{0}$ ( $\left.E_{\|}\right)$by rotating the sample holder so that the electrodes assumed the required positions. This protocol ensured that the magnetoelectric effect could be measured for (a) all orientations of the molecules with respect to $\mathbf{B}_{0}$, ranging from $z \| \mathbf{B}_{0}\left(g_{\|}\right)$to $z \perp \mathbf{B}_{0}$ $\left(g_{\perp}\right)$, and (b) for two distinct orientations with respect to $\mathbf{E}\left(\mathbf{E}_{\|}\right.$and $\left.\mathbf{E}_{\perp}\right)$, thus revealing any eventual magneto- and electroanisotropies. Figure 2 highlights the fact that in the special case of the $g_{\perp}$ resonance, the $\mathbf{E}_{\perp}$ field produces an infinite number of angles with the molecular z-axis; in all other orientations this angle is unique.

FSED spectra reveal a strong dependence, both on the electric pulse duration $t_{E}$, and on the magnetic field value, i.e. on the molecular orientation with respect to $\mathbf{B}_{0}$ (Figure 4): at $g_{\|}$the echo intensity decreases monotonically until $t_{E}=\tau=3 \mu$ s, and is fully recovered for $t_{E}=2 \tau=6 \mu$ s. At higher magnetic fields the loss of the echo intensity is more abrupt, as the molecular z-axis deviates from $\mathbf{B}_{0}$, with almost full suppression of the echo intensity at $367.5 \mathrm{mT}$ for a $3 \mu$ s pulse. Further magnetic field increases produced negative oscillations, accompanied by oscillations near $g_{\perp}$. It is quite noteworthy that for the FSED collected with $t_{E}=\tau$, the only surviving resonances are those at $g_{\|}$and $g_{\perp}$ with the latter having become negative. For $t_{E}>\tau$, the echo recoveries are fully symmetric, including the oscillatory patterns observed at $g_{\perp}$. 
The effect was also shown to be sensitive to the relative orientation of $\mathbf{E}$ with respect to $\mathbf{B}_{0}$; in particular, the decrease of the integrated echo intensity was more rapid for $\mathrm{E}_{\|}$and the oscillations at $g_{\perp}$ exhibited different frequencies.
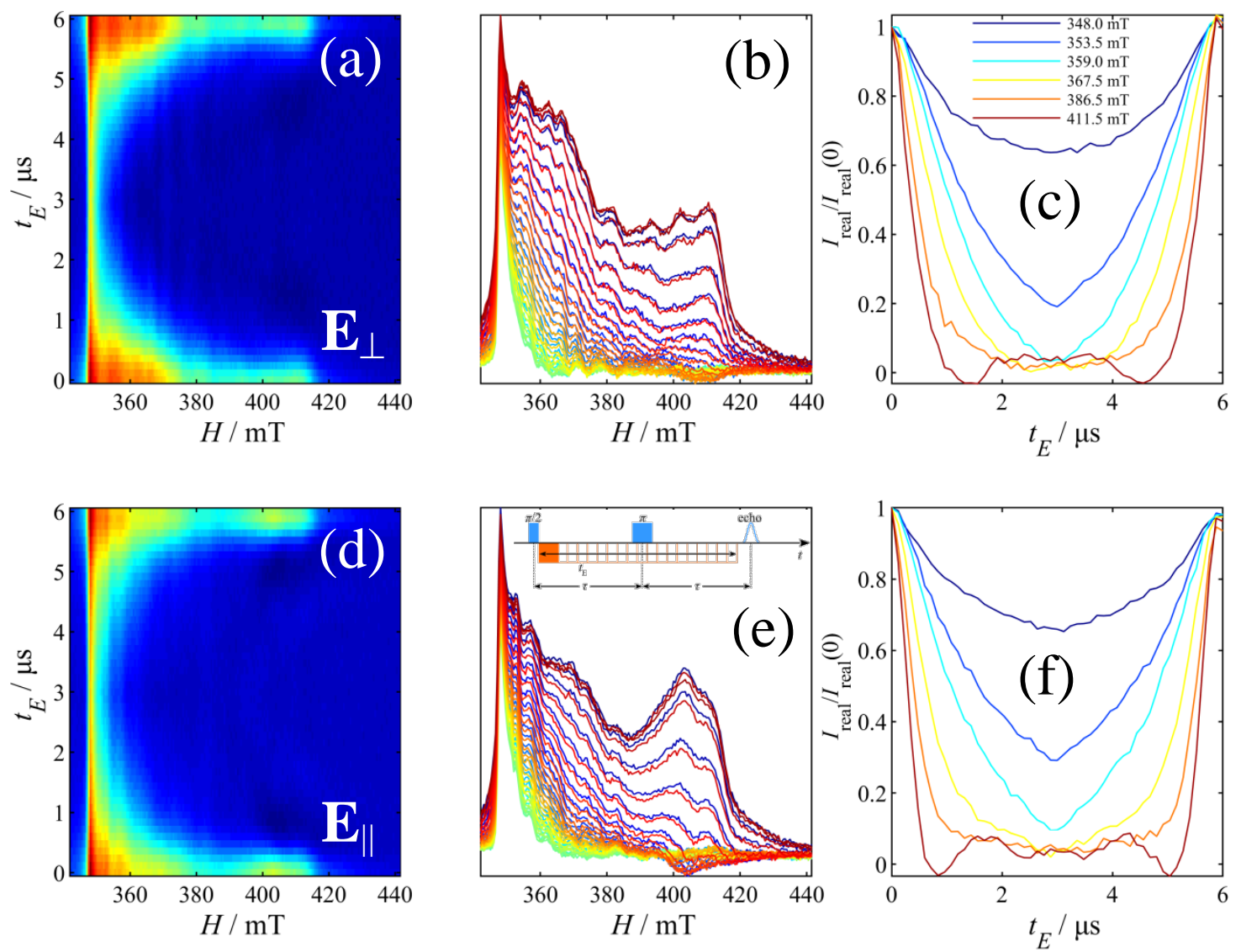

Figure 4. FSED spectra of $\mathbf{F e}_{3}(\tau=3 \mu \mathrm{s})$ implementing electric pulses after the $\pi / 2$ pulse, with incremented durations $t_{E}=0-6 \mu \mathrm{s}$. The FSED spectra are shown in 2D in panels (a) and (d) and 1D (center) representations in panels (b) and (e) for $\mathrm{E}_{\perp}$ (top) and $\mathrm{E}_{\|}$(bottom) electric fields. Panels (c) and (f) show sections of the $2 \mathrm{D}$ plots at selected magnetic fields.

The $g_{\perp}$ echo suppression, including its oscillatory behavior, was examined in greater detail for longer pulse durations ( $\tau=6 \mu \mathrm{s}$ ) and under four different electric field strengths at both electric field orientations (Figure 5). Fits to these data and Fourier-transform analyses revealed the presence of two oscillations whose frequencies increase linearly with the electric field strength (see SI). This conclusion 
is in perfect agreement with the coalescence of all curves to two families (for $E_{\|}$and $E_{\perp}$ ) when plotted to $t_{E} \times V$ (Figure 5c), confirming that the effect is proportional to the electric field strength and that it is electroanisotropic. Linear fits to the $f$ vs $|\mathbf{E}|$ curves allowed us to derive the E-field response of the oscillations (see SI), identified as the rates of frequency change $a^{k}=\mathrm{d} f / \mathrm{d} E_{k}(k=\|, \perp)$. The derived $a^{\perp} / a^{\|}$ ratios of $\sim 1.6-2.1$ indicate that the electroanisotropy of $\mathbf{F e}_{3}$, is not limited to the degree of echo suppression, but extends to the oscillatory patterns at $g_{\perp}$. We associate the appearance of two such frequencies to the electric field inhomogeneities imposed by the parallel-wire geometry. Indeed, superposition of the $\mathbf{E}_{\|}$FT spectra (Figure 5a, inset) with the histograms of $|\mathbf{E}|$ intensity distributions (Figure S1) reveals a remarkable similarity (Figure S3). This observation reinforces the E-field dependence of the oscillation frequencies, suggesting that use of flat electrodes should lead to the observation of a single frequency. 

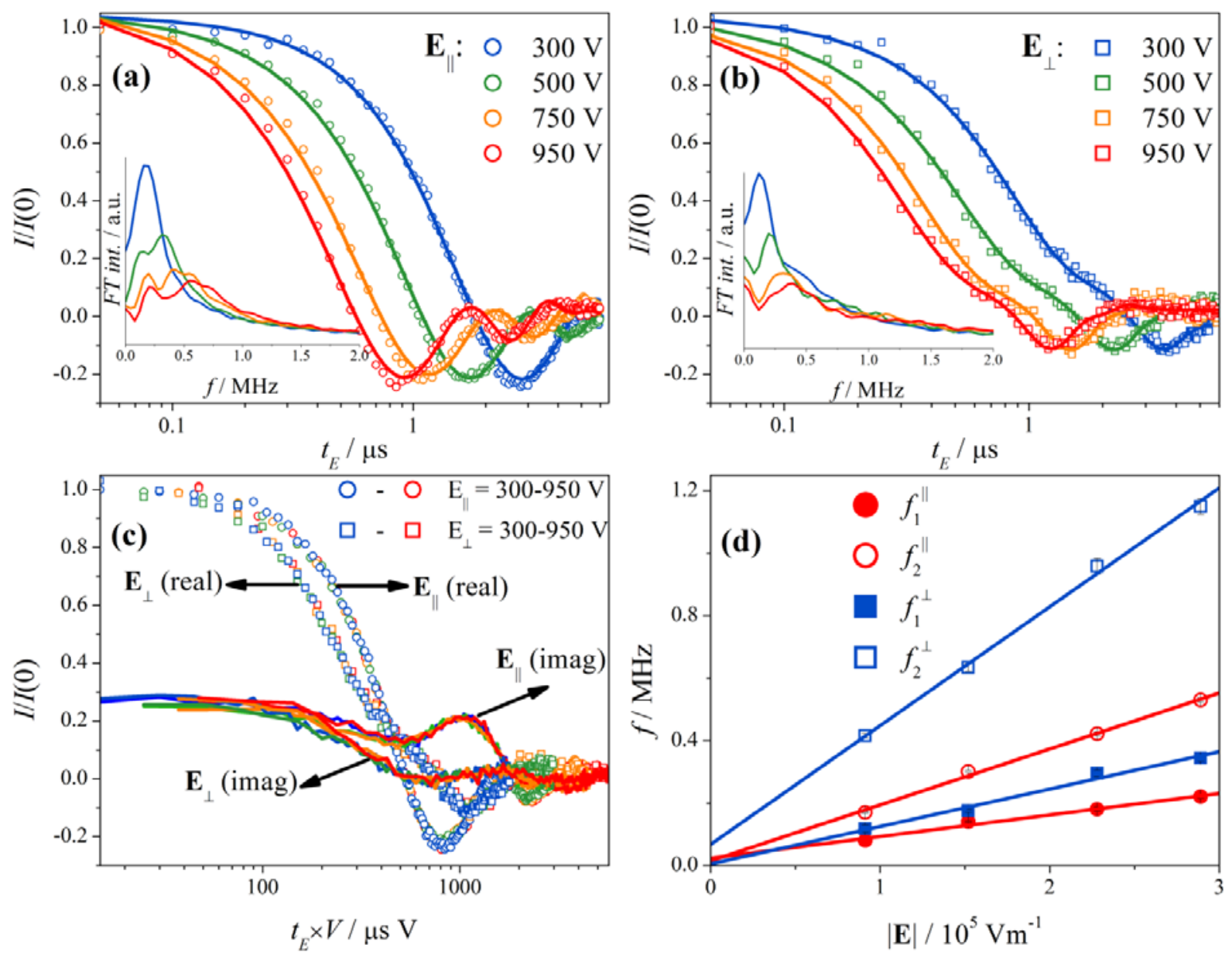

Figure 5. (a), (b) Integrated Hahn echo intensities of the $g_{\perp}$ resonance of $\mathbf{F e}_{3}$, as a function of the electric pulse intensity and duration, for $E_{\|}(a)$ and $E_{\perp}$ (b). The lines are fits to exponentially damped oscillations according to the model described in the text. The insets show the FT spectra of the oscillations for the various pulse intensities. (c) In-phase (real) and quadrature (imaginary) signals of the Hahn echo intensities plotted as a function of $t_{E} \times V$. The signals collapse into two families depending on the orientation of E. (d) Oscillation frequencies derived from the fits, plotted as a function of the average electric field intensity; the lines are linear fits.

Similar experiments revealed a drastically different behavior for $\mathbf{C r}_{3}$ (Figure 6); its echo ( $\tau=2 \mu \mathrm{s}$ ) showed a response only within the experimental error, even for $t_{E}=\tau$ (8.9\% suppression; $\sim 0.4$ standard deviations). Experiments with incremented electric pulses were similarly inconclusive, with any effect being less than one $\sigma$. FSED spectra were also collected under both electric field orientations. Although 
the much shorter $T_{2}$ relaxation times of $\mathbf{C r}_{3}$ considerably decreased the $\tau$ which would permit a reasonable $\mathrm{S} / \mathrm{N}$ ratio for the FSED spectra $(\tau=800 \mathrm{~ns})$, and hence the maximum electric pulse duration $\left(t_{E}{ }^{\max }=\tau\right)$, this should have been sufficient to reveal ME effects of similar intensity as those of $\mathbf{F e}_{3}$. However, in all cases the FSED spectra with or without electric field pulses were practically superimposable, confirming the absence of a discernible magnetoelectric coupling.
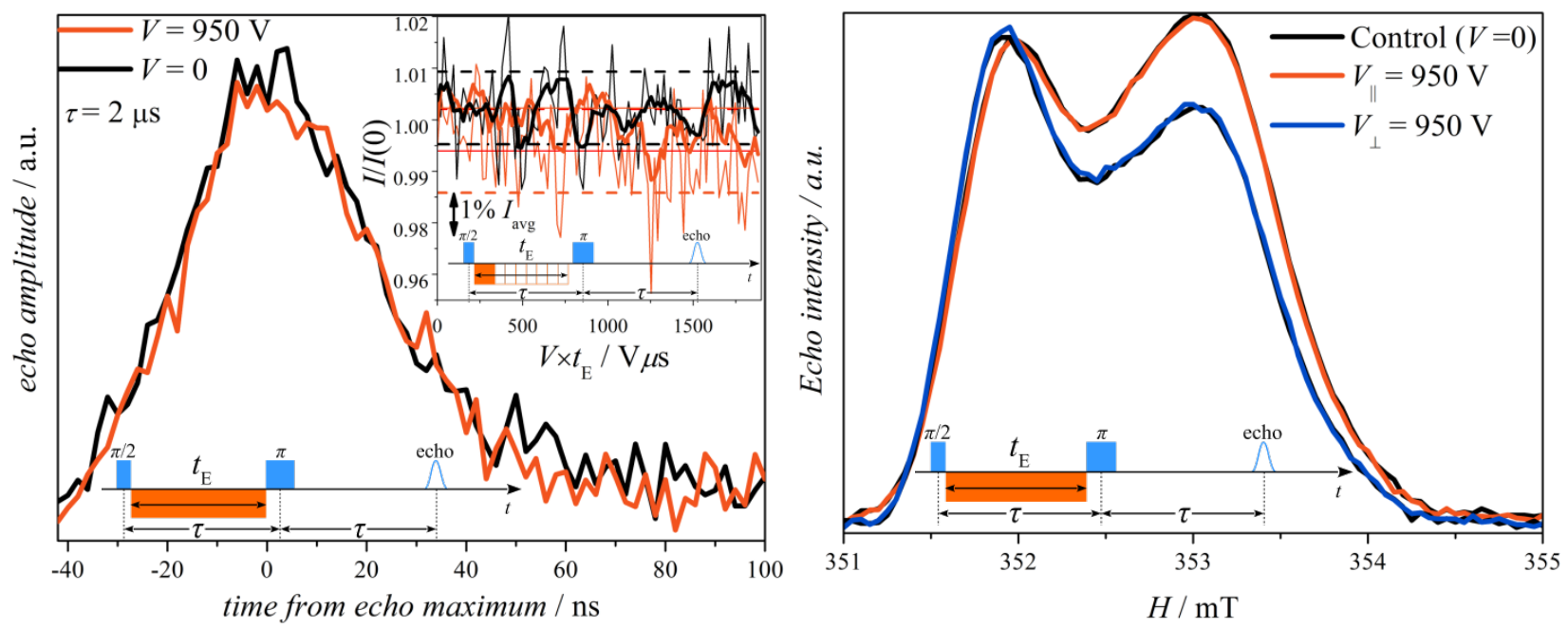

Figure 6. Effects of electric field pulses on the echo of $\mathbf{C r}_{3}$. Left: For $\tau=2 \mu$ s the effect is within the noise level. The inset shows the effect on the echo intensity for an incremented pulse. Right: FSED experiments spectra ( $\tau=800 \mathrm{~ns}$ ) carried out at both orientations of the electric field (the black lines are reference spectra recorded without electric field pulses). The differences in the FSED spectra shapes are attributed to slight distortions of the $\mathbf{B}_{1}$ map caused by the rotation of the electrodes.

These results also provide a validation of the experimental method. In particular, we considered the possibility that the magnetic fields created by the time-dependent electric fields are responsible for the observed effects. This was first addressed by experiments on TEMPOL (4-hydroxy-2,2,6,6tetramethylpiperidin-1-oxyl) which yielded no electric-field effects. More importantly, the negative results from $\mathbf{C r}_{3}$ also attest to the fact that the observed effects on $\mathbf{F e}_{\mathbf{3}}$ are intrinsic to the sample and not experimental artifacts. 
Furthermore, we considered the possibility that the effect might be caused by such magnetic fields, affecting the two complexes differently due to their different relaxation characteristics. However, in the context of the Maxwell-Ampere law, magnetic fields created by time-dependent electric flux densities (displacement currents) can only be created during the rise and fall periods of our electric field pulses. These periods are identical throughout our experiments, irrespective of the pulse duration between them. Hence if they were indeed responsible for the effect, this should be operative even for the shortest pulses of our experiments. By focusing on $\mathbf{F e}_{3}$ however, its response is a function of the pulse duration, and is minimal at the shortest pulse. This means that the magnetic fields produced during the rise and fall of the electric pulses have no discernible effect. It is the duration of the pulse, i.e. the duration of its constant part, that influences the experiment. At that period, when the electric field flux is constant, no such magnetic fields can be produced. Therefore, since the effect is a function of the pulse duration (i.e. its constant part), it can only be due to that part itself, i.e. the static electric field.

\section{Discussion}

These comparative studies provide evidence for a new mechanism giving rise to magnetoelectric coupling in MNMs. Mechanisms already known to be operative in MNMs include single-ion anisotropy, the hyperfine Stark effect and symmetric-exchange modulation, with a few theoretical models having been developed ad hoc, ${ }^{19,36,37}$ whereas extensive studies on non-molecular multiferroic materials (e.g. oxides) have detailed several such mechanisms. ${ }^{38}$ Relevant to our work, DMI has been proposed by Katsura, Nagaosa \& Balatsky (KNB model) to produce a spin current which gives rise to electric polarization, ${ }^{39}$ whereas Sergienko and Dagotto considered that DMI are induced by atomic displacements of the bridging oxides (the so-called "inverse DMI"). ${ }^{40}$

In the case of spin triangles Loss and coworkers have considered one mechanism involving transverse electric fields $\left(E_{x y}\right)$ which couple through the isotropic exchange couplings $\left(J_{i j}\right)$ through an exchange-striction model, and another involving axial electric fields $\left(E_{z}\right)$ which couple to the scalar 
spin chirality $\left(C_{z}\right)$ through spin-orbit interactions, which can actually be reduced to the DMI formalism; ${ }^{18,19}$ a quantitative theoretical prediction of the magnetoelectric coupling, considered only the former mechanism. ${ }^{21}$ Belinsky predicted a Stark effect on the spin doublets by the coupling of the vector spin chirality $\left(\kappa_{z}\right)$ to an axial electric field $\left(E_{z}\right),{ }^{36}$ also implying a DMI mechanism. Our results show that the ME coupling is highly anisotropic and easily discernible in $\mathbf{F e}_{3}$, but barely discernible in $\mathbf{C r}_{3}$. This trend correlates very well with their DMI strengths, which are 40-50 times stronger in $\mathbf{F e}_{3}$ $\left(\sim 1.7-2.0 \mathrm{~cm}^{-1}\right)^{23}$ than in $\mathbf{C r}_{3}\left(\sim 0.04 \mathrm{~cm}^{-1}\right),{ }^{24}$ and is in qualitative agreement with a semiclassical implementation of the KNB model (see SI).

We also considered an "inverse DMI", ${ }^{40}$ whereby the applied magnetic field is responsible for the development of DMI through structural distortions. We tend to discard such a mechanism based on zero-field magnetic susceptibility studies of $\mathbf{F e}_{3}$ which evidence the presence of DMI in the absence of a magnetic field (Figure S4). Similarly, we considered a symmetric-exchange mechanism like in the case of $\mathrm{Cu}^{\mathrm{II}}{ }_{3}$-saltag, ${ }^{7}$ entailing perfect frustration and nil DMI. ${ }^{41}$ However, this would be more compatible with $\mathbf{C r}_{3}\left(\left|J-J^{\prime}\right|=|\Delta J| \sim 0.3 \mathrm{~cm}^{-1} ; G_{z} \sim 0.04 \mathrm{~cm}^{-1}\right)^{24}$ than with $\mathbf{F e}_{3}\left(|\Delta J| \sim 5 \mathrm{~cm}^{-1} ; G_{z} \sim 1.7\right.$ $\left.\mathrm{cm}^{-1}\right),{ }^{23}$ meaning that it is not operative here (see SI for Hamiltonian definition). Therefore, we identify the role of DMI as the primary mechanism for the ME coupling in $\mathbf{F e}_{3}$, which would explain its absence in $\mathbf{C r}_{3}$.

A preliminary interpretation of these observations was made assuming the development of spininduced electric polarisation according to the KNB model, ${ }^{39}$ which posits that intersite polarisations are $\mathbf{P}_{i j} \propto \mathbf{e}_{i j} \times\left(\hat{\mathbf{S}}_{i} \times \hat{\mathbf{S}}_{j}\right)$, with $\mathbf{e}_{i j}$ being the unit vector connecting sites $\mathbf{M}_{i}$ and $\mathbf{M}_{j}$. The KNB model does not calculate the proportionality coefficient that allows the derivation of the absolute size of $\mathbf{P}_{i j}$, which is calculated as a unitless quantity; this is sufficient for the purpose of our qualitative comparisons. By assuming that the spin of iron(III) ions $(S=5 / 2)$ is large enough to be treated semiclassically, we used 
the local spin vectors $\mathbf{S}_{i}=\left(\left\langle\hat{S}_{x}\right\rangle,\left\langle\hat{S}_{y}\right\rangle,\left\langle\hat{S}_{z}\right\rangle\right)$ at the ground state $\alpha\left(\left\langle\hat{S}_{k}\right\rangle=\left\langle\alpha\left|\hat{S}_{k}\right| \alpha\right\rangle\right)$ to calculate intersite polarisations, and their sum $\mathbf{P}=\mathbf{P}_{12}+\mathbf{P}_{23}+\mathbf{P}_{31}$. For these calculations we considered the spin Hamiltonian (1), in which we substituted the previously determined parameters shown in Table 1. These calculations (Figure 7 and SI videos) indicate that under the influence of DMI, and for less than equilateral magnetic symmetries, a spin-induced electric polarisation is predicted when there is a $\mathbf{B}_{0}$ component within the triangle plane. Actually, the predicted $\mathbf{P}$ is maximum for $\mathbf{B}_{0} \perp z$, in line with the enhanced ME effect at $g_{\perp}$. In that case, $\mathbf{P}$ lies on the triangle plane, and its direction is uniquely described by the relative $J_{i j}$ values. In turn, $\mathbf{P}$ departs from the triangle plane only when $\left|B_{0 y}\right| \cdot\left|B_{0 z}\right| \neq 0$, where $B_{0 y}$ and $B_{0 z}$ are the $y$ - and $z$-components, respectively, of $\mathbf{B}_{0}$. Interestingly, these conclusions are in line with the observations that rotating magnetic fields can rotate the spin-current induced polarisation vectors in cycloidal magnets ${ }^{42}$ and helimagnets. ${ }^{43}$ Calculations of the norm of the total polarization vector $\mathbf{P}$ (blue vector in Figure 7) show that $|\mathbf{P}|$ vanishes when $G_{z}=0$ or when $\Delta J=0$ (Figure S5) in line with the relative ME effects of $\mathbf{F e}_{3}$ and $\mathbf{C r}_{3}$.

These simulations also allow us to proceed to a symmetry analysis of the electric field responses for the various orientations of electric and magnetic fields. Our model predicts that the strongest polarization $|\mathbf{P}|$ is generated by a magnetic field in the triangle plane $\left(\mathbf{B}_{0} \perp z\right)$, i.e. for $g_{\perp}$, which is in agreement with our experimental observations. In turn, we predict zero polarization for $\mathbf{B}_{0} \| z$, i.e. for $g_{\|}$. While the ME effect at this orientation is finite, and not zero as predicted, it still is the weakest in our series of experiments. Thus, even if our model is not quantitatively accurate, it correctly predicts the observed trend.

Regarding the orientation of the electric field, we observe that this couples to the spins when applied both along the triangle plane or normal to it. We recall that in the simple case of $g_{\perp}$, $\mathbf{P}$ will be always in-plane (see Figure 7, $B_{x}$ and $B_{y}$ panels). If we assume that $\mathbf{E}$ acts upon the spins through a torque $\boldsymbol{\tau}=$ 
$\mathbf{P} \times \mathbf{E}=|\mathrm{P}| \cdot|\mathrm{E}| \cdot \sin \theta$ on the spin-induced dipole moment, this becomes maximum when $\mathbf{E} \perp \mathbf{P}$, i.e. for an electric field normal to the triangle plane.

Nevertheless, we stress that this is a tentative rationalisation as the problem is still open; there is still important debate as to the mechanism for magnetic-exchange induced polarisations and the validity of the KNB model, ${ }^{44}$ whereas toroidal moments can also explain the magnetic-field dependent electrical polarisations. ${ }^{45}$ These issues are beyond the scope of the current study.
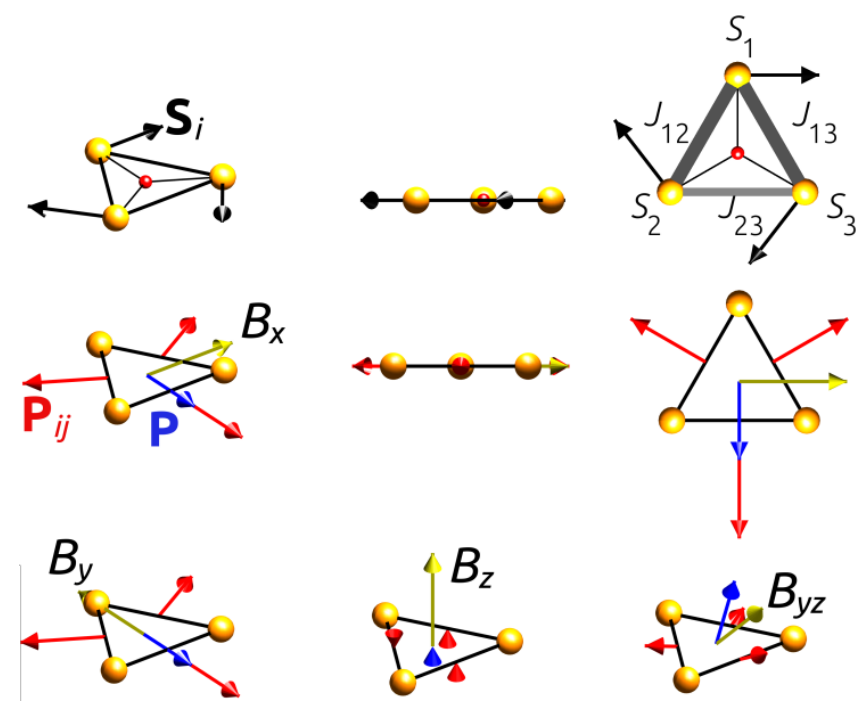

Figure 7. Top two rows. Different views of $\mathbf{F e}_{3}$ showing the spin expectation value vectors on each metal site under a magnetic field $\mathbf{B}_{0} \| x$ (top row) and the spin-induced polarisations (middle row). The model assumes $|J|>\left|J^{\prime}\right|, G_{z}>0$. Bottom row. Induced polarisations for the same magnetic model under different $\mathbf{B}_{0}$ orientations.

However, these conclusions can still have significant bearing on the question of the design of electrically controllable spin-triangle molecular spin qubits, as they outline possible predictive criteria for the development of magnetoelctric couplings. In particular, we note that for the development of spin-induced electrical polarizations, the simultaneous presence of a low magnetic symmetry and of DM interactions are required, assuming the KNB mechanism holds. A fully equilateral magnetic 
symmetry would quench the pairwise intersite polarizations, whereas in the absence of a DM term, individual spin vectors would not be misaligned so as to produce a non-zero external product.

The key parameters that determine magnetoelectric couplings under this scheme, i.e. $\Delta J$ and $\mathbf{G}$, can be determined from combined magnetic susceptometry and EPR spectroscopy studies in a clear, albeit somewhat tedious, manner. ${ }^{23,24}$ Although the precise values of such parameters need to be determined from exact calculations, a useful heuristic is the downfield shift of the $g_{\perp}$ resonance, which requires a non-zero $|\mathbf{G}|$ value, which means that when $g_{\perp} \sim g_{\|}$a magnetoelectric coupling should not be expected (like in the case of $\mathbf{C r}_{3}$ ). On the other hand, the $\Delta J$ value is not so straightforward to assess since its effect tends to counter that of G: larger $\Delta J$ values shift $g_{\perp}$ toward $g_{\|}$, whereas small $\Delta J$ values can shift it downfield, even beyond the maximum attainable magnetic field.

These parameters can also be tailored through molecular design. E.g., properly chosen ligand blends can force a low molecular symmetry that will also be reflected on the magnetic one, as has been shown in copper(II), ${ }^{46}$ or iron(III) ${ }^{47-50}$ triangles, with the latter type stabilizing $S_{\mathrm{T}}=5 / 2$ ground states in cases of extreme distortions. ${ }^{49,50}$ However, this criterion does not necessarily exclude high-symmetry structures. As a matter of fact, due to crystallographically imposed symmetry operations (a $C_{3}$ axis through the central oxide and a $\sigma_{h}$ mirror plane along the triangle plane), $\mathbf{F e}_{3}$ is a highly symmetric $\left(D_{3 h}\right)$ molecule in the solid state, in which it does exhibit a magnetoelectric coupling. ${ }^{10}$ Although these solid-state constraints are bound to relax in solution, $\mathbf{F e}_{3}$ should remain highly symmetric. Thus, its pronounced $\Delta J$ cannot be attributed to structural asymmetries, but rather to dynamic atomic vibrations as we have previously shown. ${ }^{23}$ Similarly, other high-symmetry complexes exhibit comparably favourable spin Hamiltonian parameters for the observation of a magnetoelectric coupling. ${ }^{51}$

Extending this reasoning, we may draw conclusions regarding orbital structures that may promote the breaking of magnetic symmetries through atomic vibrations. We previously commented on the 
differences of exchange interactions in $\mathbf{F e}_{3}$ and $\mathbf{C r}_{3}$, which can be correlated to the different electronic structures of the constituent ions. In particular, we argued ${ }^{24}$ that octahedral $\mathrm{Cr}^{\mathrm{III}}$ is a $\mathrm{d}^{3}$ ion with a $\left(t_{2 g}\right)^{3}\left(e_{g}\right)^{0}$ electronic configuration and empty $\mathrm{d}_{z^{2}}$ and $\mathrm{d}_{x^{2}-y^{2}}$ orbitals, while $\mathrm{Fe}^{\mathrm{III}}$ is a $\mathrm{d}^{5}$ ion with a $\left(t_{2 g}\right)^{3}\left(e_{g}\right)^{2}$ electronic configuration, with those orbitals half-filled. Since the $\mathrm{d}_{z^{2}}$ orbital in this topology is the most effective in transmitting superexchange through the central oxide, the interactions between ferric ions are stronger. This reasoning can be extended to suggest a larger role of the central oxide in producing variations of the exchange couplings through its vibrations, hence larger $\Delta J$ values. Thus, we could propose that metal ion coordination in which monatomic bridges are coordinated to half-filled metallic orbitals may induce a larger sensitivity to atomic vibrations, thus translating them to larger $\Delta J$. In fact, the low magnetic symmetries of the copper(II) complexes previously mentioned ${ }^{51}$ can be rationalized in this way, since the central monatomic bridge is coordinated through the magnetic $d_{x^{2}-y^{2}}$ orbitals.

As far as DM interactions are concerned, magnetostructural correlations and design criteria are less well developed; therefore, it is harder to predict which structures will exhibit strong DMI. E.g., complex $\mathbf{F e}_{3}$-salox (salox ${ }^{-}=$salicylaldoximate) which bears striking structural similarities with $\mathbf{F e}_{3}$ exhibits practically nil DMI. ${ }^{52}$ However, based on the reasonable assumption that DMI strengths should vary roughly in proportion to those of $\mathrm{HDvV}$ interactions, it is a reasonable assumption that monatomic superexchange pathways should play a crucial role in the transmission of sufficiently strong DM interactions. Thus, systems lacking such pathways should not be as good candidates. We may also note a general trend of chromium(III) spin triangles to exhibit very low downfield shifts of their $g_{\perp}$ resonance, indicative of weak DMI, ${ }^{24,53-56}$ which might make them less suited to exhibit strong magnetoelectric couplings. 
We now turn to the dynamic aspects of the ME effect in $\mathbf{F} \mathbf{e}_{3}$. According to the framework proposed by Mims, ${ }^{57}$ the decrease in echo intensity can be explained by a dephasing of the spin packets due to the electric pulse. An electric pulse of strength $E$ produces a phase shift $\Delta \varphi_{E}=t_{E} \Delta \omega_{E} E=2 \pi t_{E} \Delta f_{E} E$ (where $\Delta \omega_{E}$ and $\Delta f_{E}$ are the shifts in angular frequency and frequency, respectively), which can be detected by its effects on the Hahn echo decay envelope. Indeed, based on that principle, we can explain the negative echos as the result of the strong ME coupling with some spin packets (Figure S6), though the quantitative characteristics of this process are not accounted for by existing theoretical models. Mims assumed that the phase shift is additive and accumulates over successive cycles as the electric pulse duration increases. Based on this assumption, he showed that for echo decay envelopes without $(D(\tau))$ and with $\left(D_{E}(E, \tau)\right)$ the influence of electric pulses $t_{E}=\tau$, the ratio $R(E, \tau)=D_{E}(E, \tau) / D(\tau)$ is expressed as $R(E, \tau)=\exp \left(i 2 \pi \Delta f_{E} \tau E\right)$, whose real part is $\cos \left(2 \pi \Delta f_{E} \tau E\right)$. To assess this, we undertook Hahn echo decay studies for $\mathbf{E}_{\|}$and $\mathbf{E}_{\perp}$ with incremented electric pulse durations so that $t_{E}=\tau$, at several magnetic fields throughout the $g_{\|}-g_{\perp}$ range (Figure S7); in Figure 8 we show the limiting cases of $R(\tau)$ at the $g_{\|}$and $g_{\perp}$ resonances. The derived curves are characterized by a baseline which could be fitted to an arbitrarily chosen exponential decay (not shown here) and peaks corresponding to the minima of the echo decay envelopes due to ESEEM by the ${ }^{2} \mathrm{H}$ nuclei of the deuterated pyridine. The non-periodic character of the $R(\tau)$ curves indicates that the phase shift in $\mathbf{F} \mathbf{e}_{3}$ is not simply additive, as proposed by Mims, but that the magnetization undergoes saturation. The decay is stronger as we move from $g_{\|}$to $g_{\perp}$, in agreement with the increased ME effect at those resonances. 

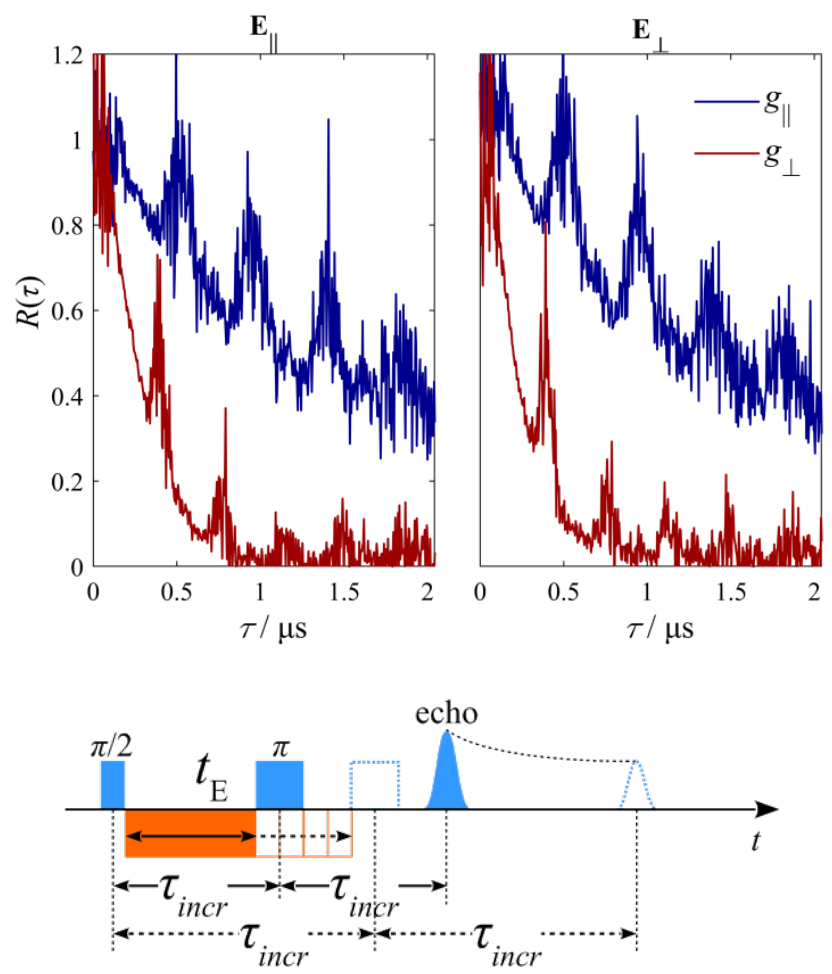

Figure 8. $R(\tau)$ ratios of $\mathbf{F e}_{3}$ for two orientations of the electric field ( $\Delta V=950 \mathrm{~V}$ ) with respect to $\mathbf{B}_{0}$ and for the $g_{\|}$and $g_{\perp}$ resonances in each case. The peaks correspond to the minima of the echo envelopes due to the ${ }^{2} \mathrm{H}$ modulations. The scheme at the bottom illustrates the pulse sequence $\left(t_{E}=\tau\right)$. The full magnetic field variation, as well as the raw Hahn echo decay envelopes are shown in Supporting Information.

Similar conclusions are reached by correlating these dephasing angles and frequencies to a putative time-invariant spin-electric coupling tensor $\tilde{\mathbf{A}}$, such that $\Delta f_{E}=2 \pi \mathbf{n} \mathbf{A} \mathbf{E}$, where $\mathbf{n}$ is the molecular magnetic axis vector. ${ }^{7}$ Using these definitions and the data from Figure 3 and Figure 5, the calculated quantities $\Delta \varphi_{E}, \Delta f_{E}$ and $A_{k}$ reveal important temporal dependencies, indicating additional dynamic effects. In particular, the asymptotic behavior of the calculated dephasing angle after long pulse durations indicates saturation effects (Figure S8).

It should be noted that like in the case of nuclear-spin qubits, slow decoherence and facile control are contradictory requirements, since strong interactions with a certain field can introduce rapid 
decoherence due to noise introduced by fluctuations of that field. Indeed, even in the absence of significant hyperfine interactions, charge noise can seriously decrease the coherence times of spin qubits experiencing strong spin-electric couplings. ${ }^{58} \mathbf{F e}_{3}$ exhibits magnetoelectric couplings whose strength, like those of other MNMs, ${ }^{7}$ are typically considered insufficient for coherently driving their spins with a microwave $\mathbf{E}_{1}$ field from standard sources, but suitable for an implementation of the " $A$ gate” proposed by Kane. ${ }^{59}$ Indeed, assuming that Mims’ framework holds for short pulses, we derive a dephasing frequency of $\sim 0.8 \mathrm{MHz}\left(|\mathbf{E}| \sim 2.9 \times 10^{5} \mathrm{Vm}^{-1}\right)$, which is approximately proportional to the electric field strength (see SI); using break junctions which can deliver electric fields of ca. $5 \times 10^{8} \mathrm{Vm}^{-}$ ${ }^{1},{ }^{60}$ a $\sim 1.4 \mathrm{GHz}$ dephasing is possible for $\mathbf{F} \mathbf{e}_{3}$, making it a viable candidate for such use.

However, spin-chirality qubits are not constrained to the microwave regime, as the transition frequencies are determined by the distance $\Delta$ of the two spin chiral states, which lies in the THz region for many transition-metal spin triangles (e.g. $\sim 56 \mathrm{~cm}^{-1}$ for $\mathbf{F e}_{3}$ ). Highly focused sources, such as $\mathrm{THz}$ pulsed lasers, can impose oscillating electric fields with $\mathbf{E}_{1}$ amplitudes close to $10^{10} \mathrm{Vm}^{-1}$, ${ }^{61}$ i.e. orders of magnitude larger than those accessible by conventional microwave sources and break junctions. In principle, these could take advantage of even moderate ME couplings to coherently drive their spins, while the qubit would remain protected from charge noise.

In conclusion, using pulsed EPR spectroscopy we have discovered a clear ME coupling in the $\mathbf{F e}_{3}$ MNM, which is characterized by unprecedented multiple anisotropies, a unique electric magnetization reversal and new dynamic phenomena not predicted by existing models. Moreover, we establish the importance of DMI and magnetic asymmetries in molecular ME materials, and we propose a semiclassical implementation of the KNB model for the first time in such materials.

\section{Supporting Information}

Electrostatic simulations (PDF)

Details of pulsed EPR experiments and fits (PDF) 
Zero-field magnetic susceptibility studies (PDF)

Simulations of spin-induced polarizations (PDF, MP4)

Spin-packet dephasing scheme (PDF)

\section{Acknowledgements}

This project has received funding from the European Union's Horizon 2020 research and innovation programme under the Marie Sklodowska-Curie grant agreement No 746060 (project "CHIRALQUBIT") and by the Fondation pour la Recherche en Chimie (Strasbourg) under the Innovation grant agreement PTu-FRC-0003 (project “MiSSTri”). We thank Dr. Anthony J. Sigillito, Dr. Mircea Trif, Dr. Serge Gambarelli, Prof. Alexander Moskvin and Prof. Daniel Loss for helpful discussions over various aspects of this work.

\section{References}

(1) Eerenstein, W.; Mathur, N. D.; Scott, J. F. Multiferroic and Magnetoelectric Materials. Nature 2006, 442 (7104), 759-765. https://doi.org/10.1038/nature05023.

(2) Pyatakov, A. P.; Zvezdin, A. K. Magnetoelectric and multiferroic media. Uspekhi Fizicheskih Nauk 2012, 182 (6), 593. https://doi.org/10.3367/UFNr.0182.201206b.0593.

(3) Bradbury, F. R.; Tyryshkin, A. M.; Sabouret, G.; Bokor, J.; Schenkel, T.; Lyon, S. A. Stark Tuning of Donor Electron Spins in Silicon. Physical Review Letters 2006, 97 (17). https://doi.org/10.1103/PhysRevLett.97.176404.

(4) Vincent, R.; Klyatskaya, S.; Ruben, M.; Wernsdorfer, W.; Balestro, F. Electronic Read-out of a Single Nuclear Spin Using a Molecular Spin Transistor. Nature 2012, 488 (7411), 357-360. https://doi.org/10.1038/nature11341.

(5) Thiele, S.; Balestro, F.; Ballou, R.; Klyatskaya, S.; Ruben, M.; Wernsdorfer, W. Electrically Driven Nuclear Spin Resonance in Single-Molecule Magnets. Science 2014, 344 (6188), 11351138. https://doi.org/10.1126/science.1249802.

(6) George, R. E.; Edwards, J. P.; Ardavan, A. Coherent Spin Control by Electrical Manipulation of the Magnetic Anisotropy. Physical Review Letters 2013, 110 (2), 027601. https://doi.org/10.1103/PhysRevLett.110.027601.

(7) Liu, J.; Mrozek, J.; Myers, W. K.; Timco, G. A.; Winpenny, R. E. P.; Kintzel, B.; Plass, W.; Ardavan, A. Electric Field Control of Spins in Molecular Magnets. Physical Review Letters 2019, 122 (3), 037202. https://doi.org/10.1103/PhysRevLett.122.037202.

(8) Ormaza, M.; Abufager, P.; Verlhac, B.; Bachellier, N.; Bocquet, M.-L.; Lorente, N.; Limot, L. Controlled Spin Switching in a Metallocene Molecular Junction. Nature Communications 2017, 8 (1). https://doi.org/10.1038/s41467-017-02151-6.

(9) Fittipaldi, M.; Cini, A.; Annino, G.; Vindigni, A.; Caneschi, A.; Sessoli, R. Electric Field Modulation of Magnetic Exchange in Molecular Helices. Nature Materials 2019, 18 (4), 329_ 334. https://doi.org/10.1038/s41563-019-0288-5. 
(10) Boudalis, A. K.; Robert, J.; Turek, P. First Demonstration of Magnetoelectric Coupling in a Polynuclear Molecular Nanomagnet: Single-Crystal EPR Studies of $\left[\mathrm{Fe}_{3} \mathrm{O}\left(\mathrm{O}_{2} \mathrm{CPh}\right)_{6}(\mathrm{Py})_{3}\right] \mathrm{ClO}_{4} \cdot$ py under Static Electric Fields. Chemistry - A European Journal 2018, 24 (56), 14896-14900. https://doi.org/10.1002/chem.201803038.

(11) Leuenberger, M. N.; Loss, D. Quantum Computing in Molecular Magnets. Nature 2001, 410 (6830), 789-793. https://doi.org/10.1038/35071024.

(12) Wernsdorfer, W.; Aliaga-Alcalde, N.; Hendrickson, D. N.; Christou, G. Exchange-Biased Quantum Tunnelling in a Supramolecular Dimer of Single-Molecule Magnets. Nature 2002, 416 (6879), 406-409. https://doi.org/10.1038/416406a.

(13) Zadrozny, J. M.; Niklas, J.; Poluektov, O. G.; Freedman, D. E. Millisecond Coherence Time in a Tunable Molecular Electronic Spin Qubit. ACS Central Science 2015, 1 (9), 488-492. https://doi.org/10.1021/acscentsci.5b00338.

(14) Godfrin, C.; Ferhat, A.; Ballou, R.; Klyatskaya, S.; Ruben, M.; Wernsdorfer, W.; Balestro, F. Operating Quantum States in Single Magnetic Molecules: Implementation of Grover's Quantum Algorithm. Physical Review Letters 2017, 119 (18), 187702.

https://doi.org/10.1103/PhysRevLett.119.187702.

(15) Bertaina, S.; Gambarelli, S.; Tkachuk, A.; Kurkin, I. N.; Malkin, B.; Stepanov, A.; Barbara, B. Rare-Earth Solid-State Qubits. Nature Nanotechnology 2007, 2 (1), 39-42. https://doi.org/10.1038/nnano.2006.174.

(16) Hussain, R.; Allodi, G.; Chiesa, A.; Garlatti, E.; Mitcov, D.; Konstantatos, A.; Pedersen, K. S.; De Renzi, R.; Piligkos, S.; Carretta, S. Coherent Manipulation of a Molecular Ln-Based Nuclear Qudit Coupled to an Electron Qubit. Journal of the American Chemical Society 2018, 140 (31), 9814-9818. https://doi.org/10.1021/jacs.8b05934.

(17) Troiani, F.; Stepanenko, D.; Loss, D. Hyperfine-Induced Decoherence in Triangular Spin-Cluster Qubits. Physical Review B 2012, 86 (16), 161409(R).

https://doi.org/10.1103/PhysRevB.86.161409.

(18) Trif, M.; Troiani, F.; Stepanenko, D.; Loss, D. Spin-Electric Coupling in Molecular Magnets. Physical Review Letters 2008, 101 (21), 217201. https://doi.org/10.1103/PhysRevLett.101.217201.

(19) Trif, M.; Troiani, F.; Stepanenko, D.; Loss, D. Spin Electric Effects in Molecular Antiferromagnets. Physical Review B 2010, 82 (4), 045429. https://doi.org/10.1103/PhysRevB.82.045429.

(20) Stepanenko, D.; Trif, M.; Tsyplyatyev, O.; Loss, D. Field-Dependent Superradiant Quantum Phase Transition of Molecular Magnets in Microwave Cavities. Semiconductor Science and Technology 2016, 31 (9), 094003. https://doi.org/10.1088/0268-1242/31/9/094003.

(21) Islam, M. F.; Nossa, J. F.; Canali, C. M.; Pederson, M. First-Principles Study of Spin-Electric Coupling in a $\left\{\mathrm{Cu}_{3}\right\}$ Single Molecular Magnet. Physical Review B 2010, 82 (15), 155446. https://doi.org/10.1103/PhysRevB.82.155446.

(22) Robert, J.; Parizel, N.; Turek, P.; Boudalis, A. K. Relevance of Dzyaloshinskii-Moriya Spectral Broadenings in Promoting Spin Decoherence: A Comparative Pulsed-EPR Study of Two Structurally Related Iron( III ) and Chromium( III ) Spin-Triangle Molecular Qubits. Physical Chemistry Chemical Physics 2019, 21 (35), 19575-19584. https://doi.org/10.1039/C9CP03422F.

(23) Georgopoulou, A. N.; Margiolaki, I.; Psycharis, V.; Boudalis, A. K. Dynamic versus Static Character of the Magnetic Jahn-Teller Effect: Magnetostructural Studies of $\left[\mathrm{Fe}_{3} \mathrm{O}\left(\mathrm{O}_{2} \mathrm{CPh}\right)_{6}(\mathrm{Py})_{3}\right] \mathrm{ClO}_{4} \cdot$ py. Inorganic Chemistry 2017, 56 (2), 762-772. https://doi.org/10.1021/acs.inorgchem.6b01912. 
(24) Boudalis, A. K.; Rogez, G.; Turek, P. Determination of the Distributions of the SpinHamiltonian Parameters in Spin Triangles: A Combined Magnetic Susceptometry and Electron Paramagnetic Resonance Spectroscopic Study of the Highly Symmetric $\left[\mathrm{Cr}_{3} \mathrm{O}(\mathrm{PhCOO})_{6}(\mathrm{Py})_{3}\right]\left(\mathrm{ClO}_{4}\right) \cdot 0.5 p y$. Inorganic Chemistry 2018, 57 (21), 13259-13269. https://doi.org/10.1021/acs.inorgchem.8b01764.

(25) Stoll, S.; Schweiger, A. EasySpin, a Comprehensive Software Package for Spectral Simulation and Analysis in EPR. Journal of Magnetic Resonance 2006, 178 (1), 42-55. https://doi.org/10.1016/j.jmr.2005.08.013.

(26) Murao, T. Jahn-Teller Effect in Trinuclear Complexes. Physics Letters A 1974, 49 (1), $33-35$. https://doi.org/10.1016/0375-9601(74)90657-4.

(27) Jones, D. H.; Sams, J. R.; Thompson, R. C. The Magnetic Behavior of Clusters of the Type $\left[\mathrm{M}_{3} \mathrm{O}(\mathrm{RCOO})_{6}\right]^{+}$. An Isotropic Model Involving Dynamic Distortions. The Journal of Chemical Physics 1984, 81 (1), 440. https://doi.org/10.1063/1.447322.

(28) Rakitin, Yu. V.; Kalinnikov, V. T.; Novotortsev, V. M. Jahn-Teller Effect in Trigonal $\mu$ Oxoclusters. Russian Chemical Bulletin 2004, 53 (11), 2478-2484. https://doi.org/10.1007/s11172-005-0142-6.

(29) Popov, A. I.; Plis, V. I.; Popkov, A. F.; Zvezdin, A. K. Jahn-Teller Effect in Multi-Spin Systems with High Exchange Interaction: Ground State Problem for V 15 Nanocluster. Physical Review B 2004, 69 (10). https://doi.org/10.1103/PhysRevB.69.104418.

(30) Rakitin, Yu. V.; Yablokov, Yu. V.; Zelentsov, V. V. EPR Spectra of Trigonal Clusters. Journal of Magnetic Resonance (1969) 1981, 43 (2), 288-301. https://doi.org/10.1016/00222364(81)90039-1.

(31) Tsukerblat, B.; Belinskii, M.; Fainzil'berg, V. Magnetochemistry and Spectroscopy of Transition Metal Exchange Clusters. Soviet Sci. Rev. B, Harwood Acad. Pub 1987, 337-482.

(32) Nishimura, H.; Date, M. Anomalous $g$-Value of a Cr-Trimer Complex, Cr-Propionate $\left\{\mathrm{Cr}_{3} \mathrm{O}(\mathrm{C}\right.$ $\left.\left.{ }_{2} \mathrm{H}_{5} \mathrm{COO}\right)_{6}\left(\mathrm{H}_{2} \mathrm{O}\right)_{3}\right\} \mathrm{NO}_{3} 2 \mathrm{H}_{2} \mathrm{O}$. Journal of the Physical Society of Japan 1985, 54 (1), 395-399. https://doi.org/10.1143/JPSJ.54.395.

(33) Honda, M.; Morita, M.; Date, M. Electron Spin Resonance in Cr-Trimer Complexes. Journal of the Physical Society of Japan 1992, 61 (10), 3773-3785. https://doi.org/10.1143/JPSJ.61.3773.

(34) Mims, W. B. Electric Field Effects in Spin Echoes. Physical Review 1964, 133 (3A), A835A840. https://doi.org/10.1103/PhysRev.133.A835.

(35) Mims, W. B. Measurement of the Linear Electric Field Effect in EPR Using the Spin Echo Method. Review of Scientific Instruments 1974, 45 (12), 1583-1591. https://doi.org/10.1063/1.1686567.

(36) Belinsky, M. I. Field-Dependent Spin Chirality and Frustration in Nanomagnets in Transverse Magnetic Field. 2. Spin Configurations, Chirality and Intermediate Spin Magnetization in Distorted Trimers. Chemical Physics 2014, 435, 95-125. https://doi.org/10.1016/j.chemphys.2013.10.009.

(37) Scarrozza, M.; Barone, P.; Sessoli, R.; Picozzi, S. Magnetoelectric Coupling and Spin-Induced Electrical Polarization in Metal-Organic Magnetic Chains. Journal of Materials Chemistry C 2016, 4 (19), 4176-4185. https://doi.org/10.1039/C5TC03613E.

(38) Tokura, Y.; Seki, S.; Nagaosa, N. Multiferroics of Spin Origin. Reports on Progress in Physics 2014, 77 (7), 076501. https://doi.org/10.1088/0034-4885/77/7/076501.

(39) Katsura, H.; Nagaosa, N.; Balatsky, A. V. Spin Current and Magnetoelectric Effect in Noncollinear Magnets. Physical Review Letters 2005, 95 (5). https://doi.org/10.1103/PhysRevLett.95.057205. 
(40) Sergienko, I. A.; Dagotto, E. Role of the Dzyaloshinskii-Moriya Interaction in Multiferroic Perovskites. Physical Review B 2006, 73 (9). https://doi.org/10.1103/PhysRevB.73.094434.

(41) Kintzel, B.; Böhme, M.; Liu, J.; Burkhardt, A.; Mrozek, J.; Buchholz, A.; Ardavan, A.; Plass, W. Molecular Electronic Spin Qubits from a Spin-Frustrated Trinuclear Copper Complex. Chemical Communications 2018, 54 (92), 12934-12937. https://doi.org/10.1039/C8CC06741D.

(42) Murakawa, H.; Onose, Y.; Kagawa, F.; Ishiwata, S.; Kaneko, Y.; Tokura, Y. Rotation of an Electric Polarization Vector by Rotating Magnetic Field in Cycloidal Magnet Eu 0.55 Y 0.45 MnO 3. Physical Review Letters 2008, 101 (19). https://doi.org/10.1103/PhysRevLett.101.197207.

(43) Ishiwata, S.; Taguchi, Y.; Murakawa, H.; Onose, Y.; Tokura, Y. Low-Magnetic-Field Control of Electric Polarization Vector in a Helimagnet. Science 2008, 319 (5870), 1643-1646.

https://doi.org/10.1126/science.1154507.

(44) Moskvin, A. S.; Drechsler, S.-L. Microscopic Mechanisms of Spin-Dependent Electric Polarization in 3 d Oxides. Physical Review B 2008, 78 (2).

https://doi.org/10.1103/PhysRevB.78.024102.

(45) Spaldin, N. A.; Fiebig, M.; Mostovoy, M. The Toroidal Moment in Condensed-Matter Physics and Its Relation to the Magnetoelectric Effect. Journal of Physics: Condensed Matter 2008, 20 (43), 434203. https://doi.org/10.1088/0953-8984/20/43/434203.

(46) Mathivathanan, L.; Boudalis, A. K.; Turek, P.; Pissas, M.; Sanakis, Y.; Raptis, R. G. Interactions between H-Bonded [ $\left.\mathrm{Cu}_{3}^{\mathrm{II}}\left(\mu_{3}-\mathrm{OH}\right)\right]$ Triangles; a Combined Magnetic Susceptibility and EPR Study. Physical Chemistry Chemical Physics 2018, 20 (25), 17234-17244. https://doi.org/10.1039/C8CP02643B.

(47) Boudalis, A. K.; Dahan, F.; Bousseksou, A.; Tuchagues, J.-P.; Perlepes, S. P. Use of the Di-2Pyridyl Ketone/3,5-Di-Tert-Butylcatechol "Blend” in Iron( III ) Chemistry: A Cationic Tetranuclear Cluster and an Anionic Trinuclear Complex. Dalton Trans. 2003, No. 17, 34113418. https://doi.org/10.1039/B305873E.

(48) Lee, Y.; Jeon, I.-R.; Abboud, K. A.; García-Serres, R.; Shearer, J.; Murray, L. J. A [3Fe-3S] ${ }^{3+}$ Cluster with Exclusively $\mu$-Sulfide Donors. Chemical Communications 2016, 52 (6), 1174-1177. https://doi.org/10.1039/C5CC07813J.

(49) Alborés, P.; Rentschler, E. A T-Shaped $\mu_{3}$-Oxido Trinuclear Iron Cluster with High Easy-Plane Anisotropy: Structural and Magnetic Characterization. European Journal of Inorganic Chemistry 2008, 2008 (25), 4004-4011. https://doi.org/10.1002/ejic.200800327.

(50) Bill, E.; Krebs, C.; Winter, M.; Gerdan, M.; Trautwein, A. X.; Flörke, U.; Haupt, H.-J.; Chaudhuri, P. A Triangular Iron(III) Complex Potentially Relevant to Iron(III)-Binding Sites in Ferreascidin. Chemistry - A European Journal 1997, 3 (2), 193-201. https://doi.org/10.1002/chem.19970030205.

(51) Ferrer, S.; Lloret, F.; Pardo, E.; Clemente-Juan, J. M.; Liu-González, M.; García-Granda, S. Antisymmetric Exchange in Triangular Tricopper(II) Complexes: Correlation among Structural, Magnetic, and Electron Paramagnetic Resonance Parameters. Inorganic Chemistry 2012, 51 (2), 985-1001. https://doi.org/10.1021/ic2020034.

(52) Raptopoulou, C. P.; Sanakis, Y.; Boudalis, A. K.; Psycharis, V. Salicylaldoxime $\left(\mathrm{H}_{2}\right.$ Salox $)$ in Iron(III) Carboxylate Chemistry: Synthesis, X-Ray Crystal Structure, Spectroscopic Characterization and Magnetic Behavior of Trinuclear Oxo-Centered Complexes. Polyhedron 2005, 24 (5), 711-721. https://doi.org/10.1016/j.poly.2005.02.002.

(53) Psycharis, V.; Raptopoulou, C. P.; Boudalis, A. K.; Sanakis, Y.; Fardis, M.; Diamantopoulos, G.; Papavassiliou, G. Syntheses, Structural, and Physical Studies of Basic Cr ${ }^{\mathrm{III}}$ and $\mathrm{Fe}^{\mathrm{III}}$ Benzilates 
and Benzoates: Evidence of Antisymmetric Exchange and Distributions of Isotropic and Antisymmetric Exchange Parameters. European Journal of Inorganic Chemistry 2006, 2006 (18), 3710-3723. https://doi.org/10.1002/ejic.200600306.

(54) Vlachos, A.; Psycharis, V.; Raptopoulou, C. P.; Lalioti, N.; Sanakis, Y.; Diamantopoulos, G.; Fardis, M.; Karayanni, M.; Papavassiliou, G.; Terzis, A. A Nearly Symmetric Trinuclear Chromium(III) Oxo Carboxylate Assembly: Preparation, Molecular and Crystal Structure, and Magnetic Properties of $\left[\mathrm{Cr}_{3} \mathrm{O}\left(\mathrm{O}_{2} \mathrm{CPh}\right)_{6}(\mathrm{MeOH})_{3}\right]\left(\mathrm{NO}_{3}\right) \cdot 2 \mathrm{MeOH}$. Inorganica Chimica Acta 2004, 357 (11), 3162-3172. https://doi.org/10.1016/j.ica.2004.04.005.

(55) Harton, A.; Nagi, M. K.; Glass, M. M.; Junk, P. C.; Atwood, J. L.; Vincent, J. B. Synthesis and Characterization of Symmetric and Unsymmetric Oxo-Bridged Trinuclear Chromium Benzoate Complexes: Crystal and Molecular Structure of [Cr3O(O2CPh)6(Py)3]ClO4. Inorganica Chimica Acta 1994, 217 (1-2), 171-179. https://doi.org/10.1016/0020-1693(93)03766-4.

(56) Ghirri, A.; van Tol, J.; Vitorica-Yrezabal, I.; Timco, G. A.; Winpenny, R. E. P. Effects of the Dzyaloshinskii-Moriya Interaction in $\mathrm{Cr}_{3}$ Triangular Spin Clusters Detected by Specific Heat and Multi-Frequency Electron Spin Resonance. Dalton Transactions 2015, 44 (31), 1402714033. https://doi.org/10.1039/C5DT01938A.

(57) Mims, W. B. The Linear Electric Field Effect in Paramagnetic Resonance; Clarendon Press: Oxford, 1976.

(58) Laird, E. A.; Pei, F.; Kouwenhoven, L. P. A Valley-Spin Qubit in a Carbon Nanotube. Nature Nanotechnology 2013, 8 (8), 565-568. https://doi.org/10.1038/nnano.2013.140.

(59) Kane, B. E. A Silicon-Based Nuclear Spin Quantum Computer. Nature 1998, 393 (6681), 133137. https://doi.org/10.1038/30156.

(60) Olavarría-Contreras, I. J.; Etcheverry-Berríos, A.; Qian, W.; Gutiérrez-Cerón, C.; CamposOlguín, A.; Sañudo, E. C.; Dulić, D.; Ruiz, E.; Aliaga-Alcalde, N.; Soler, M.; van der Zant, H. S. J. Electric-Field Induced Bistability in Single-Molecule Conductance Measurements for Boron Coordinated Curcuminoid Compounds. Chemical Science 2018, 9 (34), 6988-6996. https://doi.org/10.1039/C8SC02337A.

(61) Shalaby, M.; Hauri, C. P. Demonstration of a Low-Frequency Three-Dimensional Terahertz Bullet with Extreme Brightness. Nature Communications 2015, 6 (1). https://doi.org/10.1038/ncomms6976. 


\section{For Table of Contents Only}

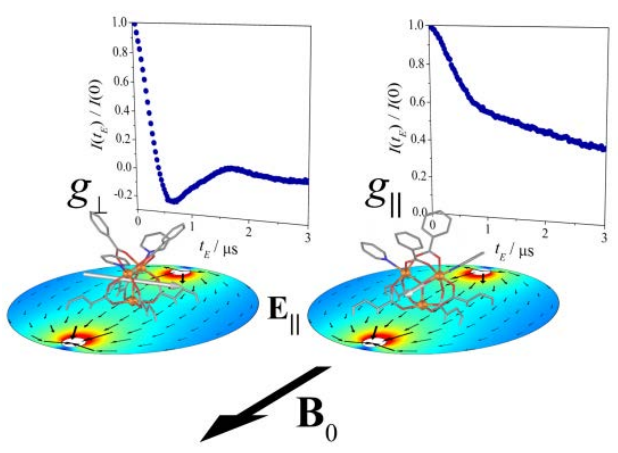

